\title{
Robust optimisation applied to the reconfiguration of distribution systems with reliability constraints
}

ISSN 1751-8687

Received on 9th December 2014 Revised on 25th September 2015 Accepted on 22nd October 2015 doi: 10.1049/iet-gtd.2015.0558 www.ietdl.org

\author{
Juan Camilo López ${ }^{1}$, Marina Lavorato ${ }^{2}$, John F. Franco ${ }^{3}$, Marcos J. Rider ${ }^{\text {凶}}$ \\ ${ }^{1}$ Department of Systems and Energy, School of Electrical and Computer Engineering, UNICAMP - University of Campinas, Campinas, \\ São Paulo, Brazil \\ ${ }^{2}$ School of Applied Science, UNICAMP - University of Campinas, Limeira, São Paulo, Brazil \\ ${ }^{3}$ Departamento de Engenharia Elétrica, Faculdade de Engenharia de Ilha Solteira, UNESP - Universidade Estadual Paulista, Ilha Solteira, \\ São Paulo, Brazil \\ $凶$ E-mail: mjrider@dsee.fee.unicamp.br
}

\begin{abstract}
This study presents a mixed-integer second-order conic programming (MISOCP) model for the robust reconfiguration of electrical distribution systems, considering the minimisation of active power losses and reliability constraints. The uncertainty at the reliability data is considered by using a linear and adjustable robust approach. The indices used to evaluate the reliability of the system are the system average interruption frequency index (SAIFI) and the system average interruption duration index (SAIDI), both of which are calculated as functions of the switch status (open or closed). Under radiality constraints, the solution generated by the proposed model is robust in terms of the reliability, i.e. the SADI and SAIFI limits imposed by regulators are not violated even if the uncertain data changes stochastically. The use of an MISOCP model guarantees convergence to optimality by using existing convex optimisation solvers. To evaluate the robustness of each solution generated by the proposed model, a set of Monte Carlo simulations were deployed. Finally, all tests were executed in a 136-node real distribution system.
\end{abstract}

\section{Nomenclature}

Sets

$\Omega_{b} \quad$ set of nodes

$\Omega_{l} \quad$ set of branches

$\Omega_{\mathrm{sw}} \quad$ set of switches

$\Omega_{z} \quad$ set of load zones

$\Omega_{z}^{\mathrm{S}} \quad$ set of source zones

\section{Parameters}

$c^{\mathrm{lss}} \quad$ cost of active power losses $[\$ / \mathrm{kW}]$

$f_{z, k}^{\mathrm{D}} \quad$ artificial flow demanded at $z \in \Omega_{z}$ for each $k \in \Omega_{b}$. Where

$\quad f_{z, k}^{\mathrm{D}}=1$ if $\hat{z}_{k}=z$, otherwise $f_{z, k}^{\mathrm{D}}=0$

$\bar{I}_{i j} \quad$ maximum current at branch $i j \in \Omega_{l}[\mathrm{~A}]$

$\bar{I}_{i j}^{\mathrm{sw}} \quad$ maximum current at switch $i j \in \Omega_{\mathrm{sw}}[\mathrm{A}]$

$l_{\text {fused }_{i j}} \quad$ Boolean parameter that indicates whether branch $i j \in \Omega_{l}$

is fuse protected $\left(l_{\text {fused }_{i j}}=1\right)$ or not $\left(l_{\text {fused }_{i j}}=0\right)$

$N_{i} \quad$ number of clients connected at node $i \in \Omega_{b}$

$P_{i}^{\mathrm{D}} \quad$ active power demanded at node $i \in \Omega_{b}[\mathrm{~kW}]$

$Q_{i}^{\mathrm{D}} \quad$ reactive power demanded at node $i \in \Omega_{b}$ [kVAr]

$r_{\mathrm{sw}} \quad$ automatic restoration time due to the coordinated operation between switching devices and the main breaker [h]

$r_{i j} \quad$ restoration time of branch $i j \in \Omega_{l}[\mathrm{~h}]$

$r_{z} \quad$ average restoration time of zone $z \in \Omega_{z}[\mathrm{~h}]$

$R_{i j} \quad$ resistance of branch $i j \in \Omega_{l}[\mathrm{~m} \Omega]$

SAIDI maximum SAIDI allowed [h/cust/year]

$\overline{\text { SAIFI }}$ maximum SAIFI allowed [fault/cust/year]

$\bar{V}$

$\underline{V}$

$\bar{X}_{i j}$ maximum voltage magnitude $[\mathrm{kV}]$ minimum voltage magnitude $[\mathrm{kV}]$ reactance of branch $i j \in \Omega_{l}[\mathrm{~m} \Omega]$
$\Gamma$

$\lambda_{i j}$

$\lambda_{\text {fused }_{k}}$

$\lambda_{z}$

$\sigma_{z}$

\section{Continuous variables}

$f_{i j, k}$

$f_{i j, k}^{+}, f_{i j, k}^{-}$

$f_{z, k}^{\mathrm{S}}$

zone of node $i \in \Omega_{b}$

zone of branch $i j \in \Omega_{l}$

impedance of branch $i j \in \Omega_{l}$, where $Z_{i j}=\sqrt{R_{i j}^{2}+X_{i j}^{2}}$ $[\mathrm{m} \Omega]$

parameter used to adjust the robustness of the proposed model

average failure rate of branch $i j \in \Omega_{l}$ [int/year]

average failure rate in the case of lateral fuse protection for each node $k \in \Omega_{b}$ [int/year]

average failure rate of zone $z \in \Omega_{z}$ [int/year]

standard deviation of the failure rate at zone $z \in \Omega_{z}$ [int/year]

\section{$I_{i j}$ \\ $I_{i j}^{\mathrm{sqr}}$ \\ $I_{i j}^{\mathrm{sw}}$ \\ $I_{i j}^{\mathrm{sw}, \mathrm{sqr}}$ \\ $p_{z, k}^{(\lambda), \omega}$}

artificial flow through switch $i j \in \Omega_{\mathrm{sw}}$, calculated for each node $k \in \Omega_{b}$

variables used by the linear equivalent of $\left|f_{i j, k}\right|$

artificial flow generated at $z \in \Omega_{z}^{\mathrm{S}}$, calculated for each node $k \in \Omega_{b}$

current magnitude through branch $i j \in \Omega_{l}[\mathrm{~A}]$

square of $I_{i j}\left[\mathrm{~A}^{2}\right]$

current magnitude through switch $i j \in \Omega_{\mathrm{sw}}[\mathrm{A}]$

square of $I_{i j}^{\mathrm{sw}}\left[\mathrm{A}^{2}\right]$

dual variable of the SAIFI constraint (48), related to the binary decision variable $\omega_{z, k}$, used in the robust formulation

$p_{z, k}^{(U), z_{\text {act }}} \quad$ dual variable of the SAIDI constraint (49), related to the binary decision variable $z_{\text {act } z k}$, used in the robust formulation

$p_{z, k}^{(U), \omega} \quad$ dual variable of the SAIDI constraint (49), related to the binary decision variable $\omega_{z, k}$, used in the robust formulation

$P_{i}^{\mathrm{S}} \quad$ active power generated at node $i \in \Omega_{b}[\mathrm{~kW}]$ 
$P_{i j} \quad$ active power flow through branch $i j \in \Omega_{l}[\mathrm{~kW}]$

$P_{i j}^{\mathrm{sw}} \quad$ active power flow through switch $i j \in \Omega_{\mathrm{sw}}[\mathrm{kW}]$

$Q_{i}^{\mathrm{S}} \quad$ reactive power generated at node $i \in \Omega_{b}[\mathrm{kVAr}]$

$Q_{i j} \quad$ reactive power flow through branch $i j \in \Omega_{l}[\mathrm{kVAr}]$

$Q_{i j}^{\mathrm{sw}} \quad$ reactive power flow through branch $i j \in \Omega_{s w}[\mathrm{kVAr}]$

SAIDI average SAIDI of the system [h/cust/year]

SAIFI average SAIFI of the system [fault/cust/year]

$U_{k} \quad$ average annual duration of interruptions at $k \in \Omega_{b}$ [h/year]

$V_{i} \quad$ voltage magnitude at node $i \in \Omega_{b}[\mathrm{kV}]$

$V_{i}^{\text {sqr }} \quad$ square of $V_{i}\left[\mathrm{kV}^{2}\right]$

$z^{(\lambda)}$ dual variable of the SAIFI constraint (48), used in the robust formulation

$z^{(U)} \quad$ dual variable of the SAIDI constraint (49), used in the robust formulation

$\Lambda_{k} \quad$ average annual failure rate at $k \in \Omega_{b}$ [int/year]

\section{Binary variables}

$y_{i j} \quad$ switch $i j \in \Omega_{s w}$ status, where $y_{i j}=1$ if switch $i j$ is closed or $y_{i j}=0$ otherwise

$z_{\text {act }_{z, k}} \quad$ binary reliability variable that identifies if zone $z \in \Omega_{z}$ is part of the upstream path between each node $k \in \Omega_{b}$ and its corresponding source, where $z_{\text {act }_{z k}}=1$ if zone $z$ is part of the upstream path; otherwise $z_{\text {act }_{z k}}=0$

$\omega_{z, k} \quad$ binary reliability variable that identifies if zone $z \in \Omega_{z}$ is at the same feeder as the node $k \in \Omega_{b}$, where $\omega_{z, k}=1$ if the zone $z$ belongs to the same feeder of $k$; otherwise $\omega_{z, k}=0$

\section{Introduction}

Electrical distribution systems (EDSs) are designed to operate economically and reliably. In this context, switching devices allocated in the feeders can be supervised and controlled in order to reduce the operational costs of the EDS and to guarantee a reliable operation. Moreover, switch operations can be used to reduce the average active power losses, to balance the load flow through feeders, or to improve the voltage profile, by modifying the topology that the system will have along a wide operational horizon, e.g. a season or a year. However, the main reasons why distribution utilities invest in switching and protection devices are to prevent permanent service interruptions and to effectively reduce the number of customers affected by faults.

Reliability measures the capacity of the network to maintain continuous operation over a defined period of time. As such, the ideal investment and operation of switching devices should optimally improve the reliability indices. Two of the most common indices used by utilities around the world are the system average interruption frequency index (SAIFI) and the system average interruption duration index (SAIDI) $[1,2]$. There are other reliability indices, such as the average energy not supplied (AENS) and the average service availability index (ASAI). However, SAIDI and SAIFI are customer-oriented indices used by many distribution regulators to measure the continuity of the electrical service and to establish reliability limits and penalties, in case of low-quality service.

Reliability indices are calculated as functions of the failure rates and the restoration times of the system components. The frequency and duration of the interruptions experienced by users can be directly related to the system's topology [3]. Eventually, reconfiguration can be used as a new strategy to maintain reliability indices below the continuity limits imposed by regulators. Thus, in addition to the classical aim of reconfiguring the EDS to minimise operational costs, it is possible to reconfigure the network in order to guarantee a reliable operation.

\subsection{Literature review}

The optimal reconfiguration problem in EDS has been extensively studied since the pioneer work of Merlin and Back [4] in 1975. A comprehensive survey on EDS reconfiguration techniques considering loss minimisation, load balancing, voltage profile improvement and service restoration can be found in [5]. However, in the last few years, the optimal reconfiguration of EDS considering reliability constraints and data uncertainty has emerged as a new EDS operating problem.

Most optimisation techniques found in the literature are based on multi-objective metaheuristic algorithms, due to the non-linear and combinatorial nature of the problem and the conflict between the objective functions. Hsiao [6] proposed a multi-objective evolution algorithm enhanced with a fuzzy technique in order to establish the system's configuration that minimises the active power losses, while improving the voltage profile, the service reliability and the number of switch operations. The reliability in [6] was determined as the capacity of the system to support unexpected loads, without considering the failure and restoration rates. Mendoza et al. [7] used a micro-genetic multi-objective algorithm for solving the problem, considering power losses minimisation and various reliability indices, such as SAIDI, SAIFI, AENS and the customer average interruption duration index. Bernardon et al. [8] proposed an heuristic methodology enhanced with a multi-criteria fuzzy algorithm for minimising the SAIFI and the total active power losses of the system, including the distribution and subtransimission losses. Amanulla et al. [9] applied a binary particle-swarm optimisation to solve the problem and used a minimal cut set algorithm to determine each node's availability index. Vitorino et al. [10] proposed an improved genetic algorithm and calculated the AENS of each configuration using non-sequential Monte Carlo simulations, based on the probability distribution of the random variables. Kavousi-Fard and Akbari-Zadeh [11] used a shuffle frog leaping algorithm enhanced with a multi-criteria fuzzy technique. The authors in [12] proposed a bat algorithm considering DG and a probabilistic load flow and, the same authors in [13] proposed a clonal selection algorithm with a probabilistic load flow and a sensitivity analysis. The failure rates in [11-13] are dependent on the current magnitudes in each line, thus, the reliability indices SAIDI, SAIFI and AENS are modified by the system's operating point.

The methodology in [14] uses a binary gravital search algorithm and calculates the energy not supplied cost, using a Weibull probability distribution to represent the random behaviour of the failure rates. Alonso et al. [15] presented an artificial immune system algorithm. The reliability in [15] is calculated with a specialised index called: power interruption equivalent frequency index, that uses a binary matrix to represent the out-of-service nodes in case of a given fault. Gupta et al. [16] proposed a genetic algorithm with weighing factors in the objective function to determine the dominance between the active power losses and the reliability indices. Narimani et al. [17] proposed an enhanced gravitational search algorithm in order to minimise the active power losses, the operational costs and to improve the system's AENS. The methodology used to calculate each node's reliability in [17] is based on the difference between the duration of the interruptions in the nodes upstream the fault and the rest of the nodes, as established by the analytical reliability assessment in [18]. Duan et al. [19] applied an enhanced genetic algorithm and reliability indices SAIDI, SAIFI, ASAI and AENS were used. The authors in [19] considered three ways that faults affect each node's reliability: first, if the fault is in another feeder; second, if the fault is in the upstream path of the node; and third, if the fault is not in the upstream path of the node.

None of the aforementioned heuristic and metaheuristic algorithms guarantee optimality of the final solution. Moreover, metaheuristic techniques are neither flexible nor easy to reproduce and modify, since most of them are based on random parameters that require trial-and-error settings, depending on the characteristics of each problem. On the other hand, mathematical models are flexible, easy to represent using mathematical programming languages, and convergence to optimality is guaranteed if the mathematical model is convex. 


\subsection{Contributions}

This paper presents a convex mixed-integer second-order conic programming (MISOCP) model for the robust reconfiguration of EDS with reliability constraints, considering uncertainty of the reliability parameters. The proposed model is used to find a system topology, i.e. the status of the switches allocated along the feeders, in order to minimise the active power losses, while avoiding the violation of the reliability indices (SAIDI and SAIFI) limits. Besides the electrical parameters, the proposed model uses the failure and restoration rates of the EDS circuits in order to analytically compute the reliability of the system. The use of the MISOCP model guarantees optimality by using classical optimisation tools.

To consider uncertainty in the reliability assessment, the model is transformed into a robust MISOCP problem. Robust models guarantee that solutions remain reliable, even if some or all, random parameters exceed the standard deviation around the mean value in accordance with the desired level of robustness. The robust approach used in this paper is based on the linear and adjustable approach presented by Bertsimas and Sim in [20]. To evaluate the level of robustness of each solution, a set of Monte Carlo simulations were conducted. The proposed model was implemented using the mathematical programming language AMPL [21], and solutions were found via the commercial optimisation solver CPLEX [22].

The main contributions of this paper are as follows:

(i) An MISOCP model for solving the reconfiguration problem of EDS with reliability indices constraints, which has the following benefits: (a) a flexible, realistic and precise model; (b) efficient computational behaviour with conventional MISOCP solvers; and (c) a convergence to optimality guaranteed by using classical optimisation techniques;

(ii) A robust programming approach that considers uncertainty of the failure rate parameters, used to evaluate the reliability indices of the EDS.

\section{Problem overview}

In this section, the general formulation of the problem will be introduced. This section also provides the characteristics of the uncertain reliability parameters used throughout this paper, i.e. the probability distribution function used to represent the uncertainty of the annual failure rates for each component

\subsection{Formulation}

The robust reconfiguration of the EDS considering reliability constraints is an operational planning problem that defines the status of the switches allocated throughout the system, in order to minimise the average active power losses, and to probabilistically avoid the violation of the reliability limits imposed by regulators. Meanwhile, all technical and operational constraints, such as voltage and current magnitude limits, substation capacities and radial operation, need to be maintained. Radiality is an operational constraint that utilities impose for technical reasons, such as simplifying protection coordination and voltage regulation, and reducing short-circuit currents.

Considering the previous comments, the objective function and the constraints of the problem are defined as follows

$$
\min _{y_{i j} \in \Omega_{\mathrm{sw}}} c^{\mathrm{lss}} \sum_{i j \in \Omega_{l}} R_{i j} I_{i j}^{\mathrm{sqr}}
$$

subject to:

$$
\text { Reconfiguration of the EDS: (7)-(19) }
$$

Reliability assessment: (22), (23) and (27)-(38)

Robust reliability constraints: $(50)-(56)$
The objective function in (1) minimises the active power losses multiplied by the cost of the active power losses $\left(c^{\text {lss }}\right)$. The set of equations in (2) determines the switch status and the AC steady-state operating point of the EDS, while guaranteeing a radial and electrically constrained operation. The assumptions and equations used to reconfigure the EDS are detailed in Section 3. The set of equations in (3) determines the SAIDI and SAIFI indices based on the switch statuses and reliability data. The hypotheses and equations used to analytically evaluate the reliability indices are shown in Section 4. Finally, the robust constraints in (4) consider the uncertainty of the reliability data and provide a reliable topology, even if the failure rates of the circuits change stochastically. The formulation of the robust constraints is detailed in Section 5 .

Note that the mathematical model presented in (1)-(4) is a mixed integer non-linear programming problem (MINLP), since the variables used to represent the switch statuses $\left(y_{i j}\right)$ are binary and the equations used to provide the $\mathrm{AC}$ steady-state operating point of the EDS in (2) are non-linear expressions. Thus, in order to solve the problem using a convex optimisation solver, such as CPLEX, the current MINLP model will be transformed into an MISOCP model, as explained in Section 3.1.

\subsection{Uncertainty characterisation}

In this paper, the failure rates of the system components are considered uncertain parameters. As shown in [3], the annual failure rates can be stochastically modelled using a Poisson probability distribution function. Then, the probability of $k$ failures in a zone $z$ is given by (5), and the standard deviation $\left(\sigma_{z}\right)$ for every stochastic parameter $\lambda_{z}$ is given by (6)

$$
\begin{gathered}
P_{z}(k)=\frac{\lambda_{z}^{k} \mathrm{e}^{-\lambda_{z}}}{k !} \quad \forall z \in \Omega_{z} \\
\sigma_{z}=\sqrt{\lambda_{z}} \quad \forall z \in \Omega_{z}
\end{gathered}
$$

In order to model the problem as a robust programming problem we need to define an interval of protection for every stochastic parameter $\lambda_{z}$. The selected interval should embrace an acceptable area of the Poisson distribution function. Since $\lambda_{z}$ cannot be negative, the chosen interval of protection for every stochastic parameter $\lambda_{z}$ is defined as $\left[\max \left\{0, \lambda_{z}-\sigma_{z}\right\}, \lambda_{z}+\sigma_{z}\right]$.

Fig. 1 shows the proposed interval of protection for $\lambda_{z}=0.25,0.5$, 0.75 and 1.0 failures/year, which are typical failure rates in practical EDS operation.

\section{Reconfiguration of the EDSs}

This section provides the mathematical foundation used for calculating the EDS steady-state operating point, considering the operation of switching devices. As shown in Fig. 2, the mathematical expressions are based on the circuit equations commonly used by the backward/ forward sweep AC load flow algorithms [23, 24] and developed according to the following hypotheses:

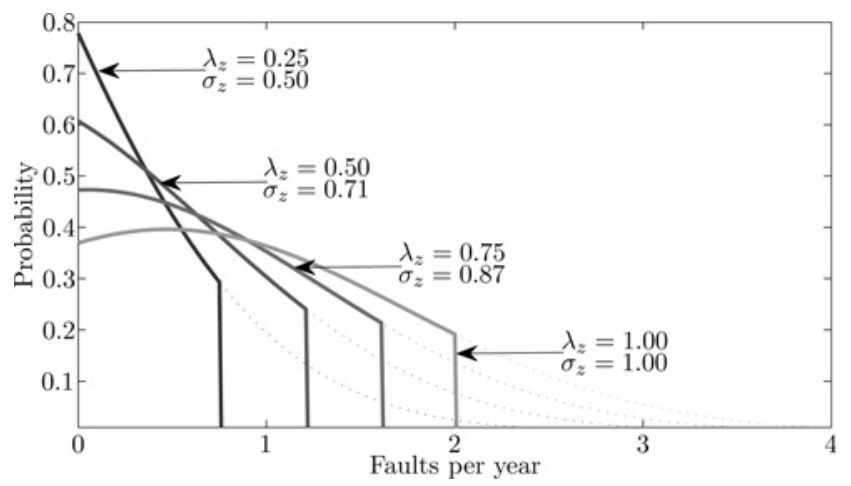

Fig. 1 Poisson distribution function for $\lambda_{z}=0.25,0.5,0.75$ and 1.0 int $/$ year 


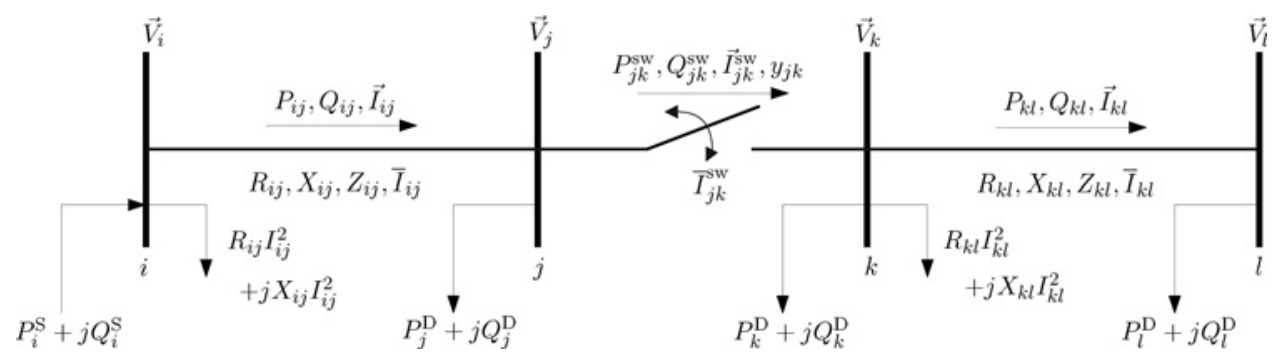

Fig. 2 EDS steady-state analysis with switching devices

(i) Electrical loads in the EDS are represented as constant active $\left(P_{i}^{\mathrm{D}}\right)$ and reactive $\left(Q_{i}^{\mathrm{D}}\right)$ power loads at every node $i \in \Omega_{b}$.

(ii) The system is assumed to be balanced and represented by its single-phase equivalent circuit. Thus, each circuit $i j \in \Omega_{l}$ has a resistance $R_{i j}$, a reactance $X_{i j}$ and a current capacity $\bar{I}_{i j}$ associated to it.

(iii) All switches $i j \in \Omega_{\mathrm{sw}}$ are considered short-length circuits with negligible impedance and limited current capacity.

The steady-state operation of EDS considering switching devices is defined by the calculation of the active $\left(P_{i}^{\mathrm{S}}\right)$ and reactive $\left(Q_{i}^{\mathrm{S}}\right)$ power generated at each source node, the voltage magnitudes at each node, the active $\left(P_{i j}, P_{i j}^{\mathrm{sw}}\right)$ and reactive $\left(Q_{i j}, Q_{i j}^{\mathrm{sw}}\right)$ power flows through each circuit and switch, and the status $\left(y_{i j}\right)$ of each switch installed in the system, in terms of the branch and node parameters. Active and reactive power losses at each circuit $i j$, given by the expression $R_{i j} I_{i j}^{2}+j X_{i j} I_{i j}^{2}$, are associated to the node $i$ in order to guarantee power balance throughout the system.

Since the current and voltage magnitudes are always presented as squared variables, hence, the following change in variables is convenient without loss of generality: $V_{i}^{\text {sqr }}=V_{i}^{2}, \quad \forall i \in \Omega_{b}$; $I_{i j}^{\mathrm{sqr}}=I_{i j}^{2}, \forall i j \in \Omega_{l}$; and $I_{i j}^{\mathrm{sqr}, \mathrm{sw}}=\left(I_{i j}^{\mathrm{sw}}\right)^{2}, \forall i j \in \Omega_{\mathrm{sw}}$. Where $V_{i}^{\mathrm{sqr}}$, $I_{i j}^{\mathrm{sqr}}$ and $I_{i j}^{\mathrm{sqr}, \mathrm{sw}}$ are continuous non-negative variables.

The sets of equations in (7) and (8) determine the voltage and the current magnitudes in the circuits as functions of the active and reactive power flows, and the system parameters

$$
\begin{gathered}
V_{i}^{\mathrm{sqr}}-V_{j}^{\mathrm{sqr}}=2\left(R_{i j} P_{i j}+X_{i j} Q_{i j}\right)+Z_{i j}^{2} I_{i j}^{\mathrm{sqr}} \quad \forall i j \in \Omega_{l} \\
V_{j}^{\mathrm{sqr}} I_{i j}^{\mathrm{sqr}}=P_{i j}^{2}+Q_{i j}^{2} \quad \forall i j \in \Omega_{l}
\end{gathered}
$$

Similarly, constraints (9) and (10) calculate the voltage and current magnitudes of the switch's nodes. Note that, since the impedances of the switches are neglected, if the switch $i j$ is closed $\left(y_{i j}=1\right)$, then both nodal voltage magnitudes will be equal; otherwise, if switch $i j$ is open $\left(y_{i j}=0\right)$, then the difference between both nodal voltages can vary freely within their operational limits

$$
\begin{gathered}
\left|V_{i}^{\mathrm{sqr}}-V_{j}^{\mathrm{sqr}}\right| \leq\left(\bar{V}^{2}-\underline{V}^{2}\right)\left(1-y_{i j}\right) \quad \forall i j \in \Omega_{\mathrm{sw}} \\
V_{j}^{\mathrm{sqr}} I_{i j}^{\mathrm{sw}, \mathrm{sqr}}=\left(P_{i j}^{\mathrm{sw}}\right)^{2}+\left(Q_{i j}^{\mathrm{sw}}\right)^{2} \quad \forall i j \in \Omega_{\mathrm{sw}}
\end{gathered}
$$

To guarantee connectivity along the feeders, (11) and (12) are used to represent the active and reactive power flow balances in the nodes, considering the power losses and the power flows of the closed switches

$$
\begin{gathered}
\sum_{j i \in \Omega_{l}} P_{j i}-\sum_{i j \in \Omega_{l}}\left(P_{i j}+R_{i j} I_{i j}^{\mathrm{sqr}}\right)+\sum_{j i \in \Omega_{\mathrm{sw}}} P_{j i}^{\mathrm{sw}}-\sum_{i j \in \Omega_{\mathrm{sw}}} P_{i j}^{\mathrm{sw}} \\
+P_{i}^{\mathrm{S}}=P_{i}^{\mathrm{D}} \quad \forall i \in \Omega_{b}
\end{gathered}
$$

$$
\begin{gathered}
\sum_{j i \in \Omega_{l}} Q_{j i}-\sum_{i j \in \Omega_{l}}\left(Q_{i j}+X_{i j} I_{i j}^{\mathrm{sqr}}\right)+\sum_{j i \in \Omega_{\mathrm{sw}}} Q_{j i}^{\mathrm{sw}} \\
-\sum_{i j \in \Omega_{\mathrm{sw}}} Q_{i j}^{\mathrm{sw}}+Q_{i}^{\mathrm{S}}=Q_{i}^{\mathrm{D}} \quad \forall i \in \Omega_{b}
\end{gathered}
$$

Constraints (13) and (14) represent the current and voltage magnitudes limits at every branch and node in the system

$$
\begin{gathered}
0 \leq I_{i j}^{\mathrm{sqr}} \leq \bar{I}_{i j}^{2} \quad \forall i j \in \Omega_{l} \\
\underline{V}^{2} \leq V_{i}^{\mathrm{sqr}} \leq \bar{V}^{2} \quad \forall i \in \Omega_{b}
\end{gathered}
$$

Constraints (15), (16) and (17) define the operational limits for the active and reactive power and current magnitudes of the switches if they are closed $\left(y_{i j}=1\right)$; if switch $i j$ is open, however, these three magnitudes are zero

$$
\begin{gathered}
\left|P_{i j}^{\mathrm{sw}}\right| \leq\left(\overline{V I}_{i j}^{\mathrm{sw}}\right) y_{i j} \quad \forall i j \in \Omega_{\mathrm{sw}} \\
\left|Q_{i j}^{\mathrm{sw}}\right| \leq\left(\overline{V I}_{i j}^{\mathrm{sw}}\right) y_{i j} \quad \forall i j \in \Omega_{\mathrm{sw}} \\
0 \leq I_{i j}^{\mathrm{sw}, \mathrm{sqr}} \leq\left(\bar{I}_{i j}^{\mathrm{sw}}\right)^{2} y_{i j} \quad \forall i j \in \Omega_{\mathrm{sw}}
\end{gathered}
$$

Constraint (18) is the analytical expression that, together with (11) and (12), guarantees the radial topology of the final solution, as demonstrated by Lavorato et al. [25]. The operator of cardinality $\Omega \mid$ in (18) determines the number of elements of the set $\Omega$

$$
\left|\Omega_{l}\right|+\sum_{i j \in \Omega_{\mathrm{sw}}} y_{i j}=\left|\Omega_{b}\right|-\left|\Omega_{b}^{S}\right|
$$

As discussed in [26], in order to avoid loop generation due to the presence of transfer nodes in the EDS, i.e. interconnection nodes with no demand, a small load of $0.001 \mathrm{~kW}$ is assumed in each transfer node so that all nodes are truly connected by (11). If, for any reason, a small load cannot be used, then an alternative mathematical approach for generating radial topologies considering interconnection nodes can be found in [27]. Finally, the binary nature of the decision variable $y_{i j}$ is established by the following equation

$$
y_{i j} \in\{0,1\} \quad \forall i j \in \Omega_{\mathrm{sw}}
$$

\subsection{MISOCP model transformation for the optimal reconfiguration of EDS}

Together, the objective function in (1) and the mathematical expression in (7)-(19) produce an MINLP problem for the optimal reconfiguration of EDS [25, 28]. However, MINLP models are not convex and modern optimisation tools cannot guarantee the optimal solution for this kind of formulation.

To achieve convexity and optimality, the equality constraints in (8) and (10) are transformed into the second-order conic 
constraints shown in the following equations

$$
\begin{gathered}
V_{j}^{\mathrm{sqr}} I_{i j}^{\mathrm{sqr}} \geq P_{i j}^{2}+Q_{i j}^{2} \quad \forall i j \in \Omega_{l} \\
V_{j}^{\mathrm{sqr}} I_{i j}^{\mathrm{sw}, \mathrm{sqr}} \geq\left(P_{i j}^{\mathrm{sw}}\right)^{2}+\left(Q_{i j}^{\mathrm{sw}}\right)^{2} \quad \forall i j \in \Omega_{\mathrm{sw}}
\end{gathered}
$$

The new MISOCP model, defined by (1), (7), (9) and (11)-(21), is a convex programming problem and, thus, can be optimally solved via branch and bound techniques implemented by classical optimisation tools.

If the new MISOCP model has a feasible solution, and the dual variables related to constraints (20) and (21) are greater than zero, then the solution provided by the MISOCP model is the optimal solution of the original MINLP model, as demonstrated by Franco et al. [29] for the EDS expansion planning problem.

\section{Reliability assessment}

This section presents the formulation used to calculate the reliability indices, SAIDI and SAIFI, and the hypotheses made for computing the reliability in terms of the system's topology.

To relate the reliability assessment to the binary decision variable $y_{i j}$, is necessary to add a new set of constraints to the reconfiguration model presented in the previous section. The procedure used to define the reliability indices of EDS in this paper is based on the fundamental analysis for radial networks considering switching devices and fuses, described by Billinton and Allan [3]. The reliability of EDS is defined by the following indices

$$
\begin{aligned}
& \text { SAIFI }=\frac{\sum_{k \in \Omega_{b}} \Lambda_{k} N_{k}}{\sum_{k \in \Omega_{b}} N_{k}} \\
& \text { SAIDI }=\frac{\sum_{k \in \Omega_{b}} U_{k} N_{k}}{\sum_{k \in \Omega_{b}} N_{k}}
\end{aligned}
$$

The SAIFI in (22) indicates how often average users suffer from a sustained interruption in a year, and is based on the annual failure rate $\left(\Lambda_{k}\right)$ of every node $k \in \Omega_{b}$. SAIDI in (23) indicates the average duration of the interruptions experienced by users in a year, and is based on the annual duration of the interruptions $\left(U_{k}\right)$ of every node $k \in \Omega_{b}$. Normally $\Lambda_{k}$ and $U_{k}$ are determined by the statistical or predictive value of the annual failure rate and restoration time of the EDS components. However, protection devices, such as fuses, reclosers and sectionalisers installed along feeders, complicate the reliability assessment. Thus, this section presents a set of constraints to analytically calculate the SAIFI and SAIDI according to the system's topology, considering the operation of switching devices and fuses in EDS.

\subsection{Pre-processing procedure}

Before formulating the reliability constraints, the following pre-processing steps need to be carried out:

Step 1: Define the set of zones $\left(\Omega_{z}\right)$ : Each zone is a section of the EDS, in which the nodes are radially connected and delimited by switching devices. In effect, all of the users that belong to the same zone would experience the same frequency and duration of interruptions in a year. Thus, nodes are aggregated into zones in order to simplify the reliability assessment

Step 2: Calculate parameters $\lambda_{z}$ and $r_{z}$ for all of the zones: Expressions (24) and (25) calculate the average failure rate $\left(\lambda_{z}\right)$ and average restoration time $\left(r_{z}\right)$, respectively, for each zone $z$ defined in Step 1.

$$
\begin{aligned}
& \lambda_{z}=\sum_{\substack{i j \in \Omega_{\hat{z}_{l}} \\
i j \text { is not fuse } \\
\text { protected }}} \lambda_{i j} \quad \forall z \in \Omega_{z} \\
& r_{z}=\frac{1}{\left|\Omega_{\hat{z}_{l}}\right|} \sum_{i j \in \Omega_{\hat{z}_{l}}} r_{i j} \quad \forall z \in \Omega_{z}
\end{aligned}
$$

Set function $\Omega_{\hat{z}_{l}}=\left\{i j \in \Omega_{l} \mid \hat{z}_{i j}=z\right\}$ contains all of the branches that belong to the specific zone $z$.

$$
\begin{aligned}
\lambda_{\text {fused }_{k}}= & \begin{cases}\sum_{i j \in \Omega_{l}} \lambda_{i j} & \text { If } l_{\text {fused }_{i j}}=1, \text { and } k, i \text { and } j \text { belong to the same path } \\
0 & \text { Otherwise }\end{cases} \\
& \forall k \in \Omega_{b}
\end{aligned}
$$

Parameter $\lambda_{\text {fused }_{k}}$ makes it possible to distinguish fuse-protected nodes in lateral circuits from those that belong to the main feeder in each zone. The conditional summation in (26) considers the downstream embracing effect of fuses, which protects circuits from sustained faults downstream of the point where the fuse is installed.

\subsection{Hypotheses}

The following hypotheses form the basis of the proposed reliability assessment:

(i) Faults are considered sustained short circuits, therefore, every fault in a non-fuse-protected circuit of the EDS will trigger a main breaker operation.

(ii) Permanent faults in lateral fuse-protected circuits are isolated due to the fuse blow [see (26)].

(iii) Fuses located in the main feeder of each zone are disregarded from this analysis.

(iv) Faults in one feeder do not affect the other feeders.

(v) Switching devices, coordinated with the main breaker operation, produce the quick isolation of the zones that do not belong to the upstream path of a node whose reliability is being evaluated. In other words, if a fault occurs in a zone that does not belong to the upstream path of the node whose reliability is being evaluated, the restoration scheme will restore the interruption in at least $r_{\mathrm{sw}}$ time.

As an example, consider the 16-node radial system shown in Figs. $3 a$ and $b$. If the node whose reliability is being evaluated is node 10 , then the upstream path in Fig. $3 a$ is formed by zones 4 and 1 (shaded areas), and all interruptions due to faults in the rest of the zones, i.e. zones 2, 3 and 5, are quickly restored using the coordinated operation of the switches. Otherwise, if the fault is located in any of the upstream zones, then it would take a nominal restoration time to repair the interruption. Thus, the frequency and duration of the interruptions of node 10 shown in Fig. $3 a$ are

$$
\begin{gathered}
\Lambda_{10(a)}=\lambda_{F 1}+\lambda_{Z 1}+\lambda_{Z 3}+\lambda_{Z 4} \\
U_{10(a)}=\lambda_{F 1} r_{F 1}+\lambda_{Z 1} r_{Z 1}+\lambda_{Z 4} r_{Z 4}+\lambda_{Z 3} r_{\mathrm{sw}}
\end{gathered}
$$

Note that, as shown in Fig. $3 b$, if the topology of the system changes, then the upstream path of node 10 also changes, and the reliability experienced by the users connected to node 10 is different from the one shown in Fig. $3 a$. The frequency and duration of the 


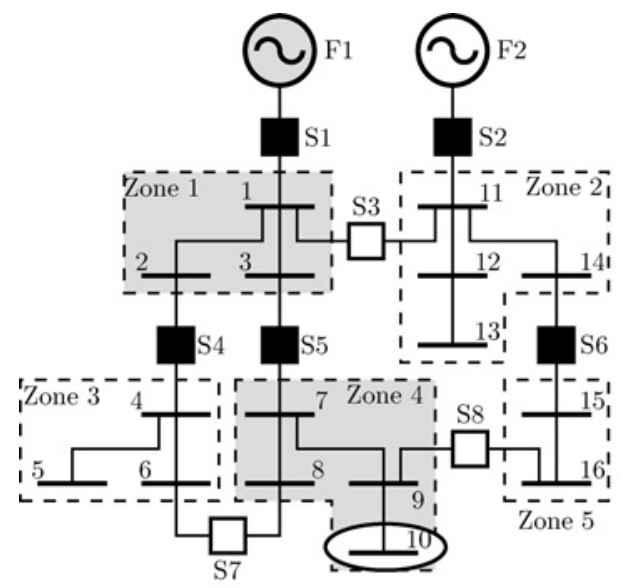

a

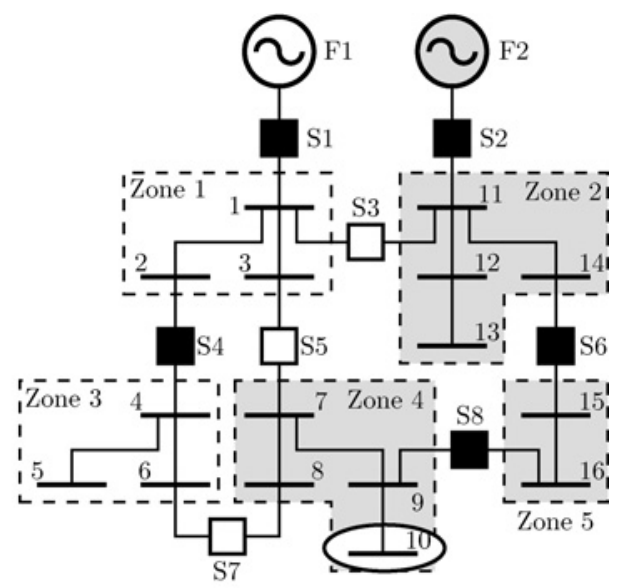

$b$

Fig. 3 Hypotheses 5. Influence of the system's topology in the reliability assessment

$a$ Upstream path of the node 10 is formed by zones 1 and 4

$b$ Upstream path of the node 10 is formed by zones 2,4 and 5

interruptions of node 10 shown in Fig. $3 b$ are

$$
\begin{gathered}
\Lambda_{10(b)}=\lambda_{F 2}+\lambda_{Z 2}+\lambda_{Z 4}+\lambda_{Z 5} \\
U_{10(b)}=\lambda_{F 2} r_{F 2}+\lambda_{Z 2} r_{Z 2}+\lambda_{Z 4} r_{Z 4}+\lambda_{Z 5} r_{Z 5}
\end{gathered}
$$

\subsection{Reliability constraints}

The set of constraints (27)-(38) is a mathematical adaptation of the procedure proposed in [3] to analytically estimate the reliability of radial networks, considering switching devices, fuse operation and the hypotheses established in the prior subsection

$$
\begin{aligned}
& \sum_{\substack{j i \in \Omega_{\mathrm{sw}} \\
\hat{z}_{i}=z}} f_{j i, k}-\sum_{\substack{i j \in \Omega_{\mathrm{sw}} \\
\hat{z}_{i}=z}} f_{i j, k}+f_{z, k}^{\mathrm{s}}=f_{z, k}^{\mathrm{D}} \quad \forall k \in \Omega_{b}, \forall z \in \Omega_{z} \\
& \left|f_{i j, k}\right| \leq y_{i j} \quad \forall i j \in \Omega_{\mathrm{sw}}, \forall k \in \Omega_{b} \\
& z_{\text {act } \hat{z}_{j}, k} \geq\left|f_{i j, k}\right| \quad \forall i j \in \Omega_{\text {sw }}, \forall k \in \Omega_{b} \\
& z_{\text {act } \hat{i}_{i}, k} \geq\left|f_{i j, k}\right| \quad \forall i j \in \Omega_{\mathrm{sw}}, \forall k \in \Omega_{b} \\
& \omega_{\hat{z}_{j}, k} \geq \omega_{\hat{z}_{i}, k}+y_{i j}-1 \quad \forall i j \in \Omega_{\mathrm{sw}}, \forall k \in \Omega_{b} \\
& \omega_{\hat{z}_{i}, k} \geq \omega_{\hat{z}_{j}, k}+y_{i j}-1 \quad \forall i j \in \Omega_{\mathrm{sw}}, \forall k \in \Omega_{b} \\
& z_{\text {act } z, k} \leq \omega_{z, k} \quad \forall k \in \Omega_{b}, \forall z \in \Omega_{z} \\
& \omega_{z, k} \leq f_{z, k}^{\mathrm{S}} \quad \forall k \in \Omega_{b}, \forall z \in \Omega_{z}^{\mathrm{S}} \\
& z_{\text {act } z, k} \leq \sum_{\substack{i j \in \Omega_{\text {sw }} \\
\hat{z}_{i}=z}}\left|f_{i j, k}\right|+\sum_{\substack{j i \in \Omega_{\text {sw }} \\
\hat{z}_{i}=z}}\left|f_{j i, k}\right| \quad \forall k \in \Omega_{b}, \forall z \in \Omega_{z} \\
& \Lambda_{k}=\sum_{z \in \Omega_{z}} \omega_{z, k} \lambda_{z}+\lambda_{\text {fused }_{k}} \quad \forall k \in \Omega_{b} \\
& U_{k}=\sum_{z \in \Omega_{z}}\left[z_{\mathrm{act}_{z, k}} \lambda_{z} r_{z}+\left(\omega_{z, k}-z_{\mathrm{act}_{z, k}}\right) \lambda_{z} r_{\mathrm{sw}}\right]+\lambda_{\text {fused }_{k}} r_{\hat{z}_{k}} \\
& \forall k \in \Omega_{b} \\
& z_{\text {act }_{z, k}}, \omega_{z, k} \in\{0,1\} \quad \forall k \in \Omega_{b}, \forall z \in \Omega_{z}
\end{aligned}
$$

Since the reliability indices, SAIFI and SAIDI, depend on the system's topology, (27)-(38) define the annual failure rate of every node $\left(\Lambda_{k}\right)$, and the annual duration of the interruptions of every node $\left(U_{k}\right)$, according to the value of the binary decision variable $y_{i j}$.

Constraint (27) represents an artificial flow balance throughout the zones and the switches of the system. The continuous variable $f_{i j, k}$ is an artificial flow that identifies the upstream path between each node $k$ and its corresponding source, where $i j \in \Omega_{\mathrm{sw}}$. The identification of the upstream path is necessary in order to evaluate the average annual outage time $\left(U_{k}\right)$ for each node, as established by the fifth hypotheses in Section 4.2. Since parameter $f_{z, k}^{\mathrm{D}}$ is equal to 1 if $\hat{z}_{k}=z, 0$ otherwise; constraint (27) is the analytical formulation that finds the shortest path through a radial graph between each node $k$ and its corresponding source [30].

To maintain linearity, the artificial flow is represented by the linear set (39)-(41). The absolute value of $f_{i j, k}$ is replaced by its linear equivalent, $\left|f_{i j, k}\right|=f_{i j, k}^{+}+f_{i j, k}^{-}$, in all equations

$$
\begin{gathered}
f_{i j, k}=f_{i j, k}^{+}-f_{i j, k}^{-} \quad \forall i j \in \Omega_{\mathrm{sw}} \forall k \in \Omega_{b} \\
f_{i j, k}^{+} \geq 0 \quad \forall i j \in \Omega_{\mathrm{sw}} \forall k \in \Omega_{b} \\
f_{i j, k}^{-} \geq 0 \quad \forall i j \in \Omega_{\mathrm{sw}} \forall k \in \Omega_{b}
\end{gathered}
$$

Constraint (28) links the artificial flow $f_{i j, k}$ with the binary decision variables $y_{i j}$. If switch $i j$ is open $\left(y_{i j}=0\right)$, then (28) forbids flow through it; otherwise, constraints (27) decides if switch $i j$ is part of the upstream path of the node $k$.

The binary reliability variables $z_{\text {act } z, k}$ and $\omega_{z, k}$ are defined by (29), (30) and (31), (32), respectively. $z_{\text {actz }, k}$ determines the zones that are part of the upstream path between each node $k$ and its corresponding source. If the artificial flow through the switch $i j$ is positive, then (29) and (30) establish that both zones, $\hat{z}_{i}$ and $\hat{z}_{j}$, are part of the upstream path of the node $k$. On the other hand, $\omega_{z, k}$ determines if the zone $z$ and the node $k$ belong to the same feeder, i.e. if both are supplied by the same source. If the switch $i j$ is closed, and whether $\hat{z}_{i}$ or $\hat{z}_{j}$ is supplied by the same source than $k$, then (31) and (32) establish that both zones belong to the same feeder of $k$.

Constraints (33)-(35) establish the following set of trivial relationships between the binary decision variables $z_{\text {actz }, k}$ and $\omega_{z, k}$ and the artificial flow $f_{i j, k}$ :

(i) Constraint (33) defines that if the zone $z$ does not belong to the same feeder of a node $k$, eventually the zone cannot be part of the upstream path, thus if $\omega_{z, k}=0$, then $z_{\text {act } z, k}=0$.

(ii) Constraint (34) establishes that if no artificial flow is generated at substation zone $z \in \Omega_{z}^{S}$ for a given node $k$, it means that $k$ does not belong to the feeder supplied by $z$, thus if $f_{z, k}^{\mathrm{s}}=0$, then $\omega_{z, k}=0$. 
(iii) Constraint (35) determines that $z_{\text {act }, k}=0$, if all artificial flows associated to the zone $z$ are zero, for a given node $k$.

Constraints (33)-(35) avoid inconsistencies between the binary variables and enhance the accuracy of the final solution produced by the solver.

Equation (36) defines the average annual failure rate $\left(\Lambda_{k}\right)$ at every node, while (37) defines the average annual duration of interruptions $\left(U_{k}\right)$ at every node. Both variables are functions of the average failure rates $\left(\lambda_{z}, \lambda_{\text {fused } k}\right)$, the restoration times $\left(r_{z}, r_{\mathrm{sw}}\right)$ and the binary reliability variables $z_{\text {act } z, k}$ and $\omega_{z, k}$. The reliability indices are assessed by (22) and (23), where $N_{k}$ is the number of customers connected to the node $k$.

Finally, constraints (42) and (43) can be appended to the mathematical model in order to guarantee that the average SAIDI and SAIFI do not violate the limits imposed by regulators

$$
\begin{gathered}
\text { SAIFI } \leq \overline{\text { SAIFI }} \\
\text { SAIDI } \leq \overline{\text { SAIDI }}
\end{gathered}
$$

The set of constraints in (27)-(43) is a mixed-integer linear equation system that can be added to the reconfiguration MISOCP problem in (1), (7), (9) and (11)-(23) in order to determine and limit the reliability of the final solution. The new reconfiguration problem with reliability constraints is a convex programming problem that can be solved with commercial solvers, such as CPLEX. The solution produced by the proposed reconfiguration model obtains the EDS's topology that minimises active power losses and guarantees that the average reliability indices, SAIFI and SAIDI, remain below their limits.

\section{Robust reliability constraints}

A major disadvantage of the proposed reliability constraints in (27)(43) is the fact that reliability parameters, such as annual failure rates, are stochastic, uncontrollable and highly uncertain parameters. Thus, in practice, the use of average values of $\lambda_{z}$ can lead to unrealistic and unreliable solutions. Accordingly, this section considers the uncertainty characterisation of the reliability parameters $\lambda_{z}$ shown in Section 2.2 in order to transform the deterministic reliability constraints (42) and (43) into robust reliability constraints.

\subsection{Linear robust optimisation}

Robust optimisation allows models to remain feasible even if some or all, random parameters change during the application of the solution produced by the robust model. A linear and adjustable approach for turning deterministic models into robust models was presented by Bertsimas and Sim [20].

Consider the nominal optimisation problem given by the following equation

$$
\left\{\begin{array}{l}
\min \sum_{j} c_{j} x_{j} \\
\text { s.t. } \\
\sum_{j} a_{i j} x_{j} \leq b_{i} \quad \forall i \\
l_{j} \leq x_{j} \leq u_{j} \quad \forall j
\end{array}\right.
$$

where $x_{j}$ is the set of decision variables, $c_{j}$ is the set of costs at the objective function and $a_{i j}$ is the set of coefficients at every constraint $i$. Consider that $\Omega_{U i}$ contains all of the coefficients in the $i$ th constraint that are subject to uncertainty. If every uncertain coefficient $a_{i j}, \forall j \in \Omega_{U i}$ is modelled as a random variable that takes values within the symmetrically bounded interval $\left[a_{i j}-\hat{a}_{i j}, a_{i j}+\hat{a}_{i j}\right]$, then the robust formulation presented in [20] is given by the following equation

$$
\left\{\begin{array}{l}
\min \sum_{j} c_{j} x_{j} \\
\text { s.t. } \\
\sum_{j} a_{i j} x_{j}+z_{i} \Gamma_{i}+\sum_{j \in \Omega_{U i}} p_{i j} \leq b_{i} \quad \forall i \\
z_{i}+p_{i j} \geq \hat{a}_{i j}\left|x_{j}\right| \quad \forall i, \forall j \in \Omega_{U i} \\
l_{j} \leq x_{j} \leq u_{j} \quad \forall j \\
p_{i j} \geq 0 \quad \forall i, \forall j \in \Omega_{U i} \\
z_{i} \geq 0 \quad \forall i
\end{array}\right.
$$

The new robust model in (45) remains linear since the term $\left|x_{j}\right|$ is easy to linearise, and a new set of continuous variables $z_{i}$ and $p_{i j}$, $\forall i, \forall j \in \Omega_{U i}$ is added. Parameter $\Gamma_{i}$ adjusts the level of robustness of every constraint $i$ in the final solution. If $\Gamma_{i}=0$, then constraint $i$ will not be robust, and its coefficients are expected to be deterministic parameters $\left(\hat{a}_{i j}=0\right)$. Otherwise, if $\Gamma_{i}=\left|\Omega_{U i}\right|$, then constraint $i$ will be totally protected, even if all of the random coefficients of $i$ reach their known limits. Although, the last scenario is a tempting protective approach, it would lead to an extremely conservative solution, with the worst objective function possible. The mathematical development used to transform nominal deterministic mathematical models into robust programming problems is detailed in the Appendix.

\subsection{Robust SAIDI and SAIFI limits}

Let (42) and (43) be the constraints that limit the reliability indices, SAIDI and SAIFI, subject to the uncertainty of parameter $\lambda_{z}$. SAIFI and SAIDI in (42) and (43) are replaced by their equivalent (22) and (23) to obtain the following equations

$$
\begin{aligned}
& \frac{\sum_{k \in \Omega_{b}} \Lambda_{k} N_{k}}{\sum_{k \in \Omega_{b}} N_{k}} \leq \overline{\text { SAIFI }} \\
& \frac{\sum_{k \in \Omega_{b}} U_{k} N_{k}}{\sum_{k \in \Omega_{b}} N_{k}} \leq \overline{\text { SAIDI }}
\end{aligned}
$$

$\Lambda_{k}$ and $U_{k}$ in (46) and (47) are replaced by their equivalents given by (36) and (37) for each node $k \in \Omega_{b}$ to obtain the following equations

$$
\begin{aligned}
& \sum_{k \in \Omega_{b}} \sum_{z \in \Omega_{z}} N_{k} \lambda_{z} \omega_{z, k} \leq \sum_{k \in \Omega_{b}} N_{k}\left(\overline{\mathrm{SAIFI}}-\lambda_{\text {fused } k}\right) \\
& \sum_{k \in \Omega_{b}} \sum_{z \in \Omega_{z}}\left[N_{k}\left(r_{z}-r_{\mathrm{sw}}\right) \lambda_{z} z_{\text {act } k, z}+N_{k} r_{\mathrm{sw}} \lambda_{z} \omega_{z, k}\right] \\
& \leq \sum_{k \in \Omega_{b}} N_{k}\left(\overline{\mathrm{SAIDI}}-\lambda_{\text {fused } k} r_{\hat{z}_{k}}\right)
\end{aligned}
$$

Note that (48) and (49) have the form $\sum_{j} a_{i j} x_{j} \leq b_{i}$. The decision variables are the binary reliability variables $z_{\text {act } k, z}$ and $\omega_{z, k}$, and all coefficients are multiplied by the random parameter $\lambda_{z}$. Also, the right-side members of (48) and (49) are constants factors.

As explained in Section 2.2, the protection interval of the random parameter $\lambda_{z}$ is bounded by the term $\left[\lambda_{z}-\sigma_{z}, \lambda_{z}+\sigma_{z}\right]$. Thus, the coefficients that multiplied the binary variables $z_{\text {act } k, z}$ and $\omega_{z, k}$ in (48) and (49) are bounded by the same protection interval. Applying the linear robust formulation given by (45), then (48) and (49) are transformed into robust constraints given by the following equations

$$
\begin{aligned}
& \sum_{k \in \Omega_{b}} \sum_{z \in \Omega_{z}} N_{k} \lambda_{z} \omega_{z, k}+z^{(\lambda)} \Gamma+\sum_{k \in \Omega_{b}} \sum_{z \in \Omega_{z}} p_{z, k}^{(\lambda), \omega} \\
& \leq \sum_{k \in \Omega_{b}} N_{k}\left(\overline{\mathrm{SAIFI}}-\lambda_{\text {fused } k}\right)
\end{aligned}
$$




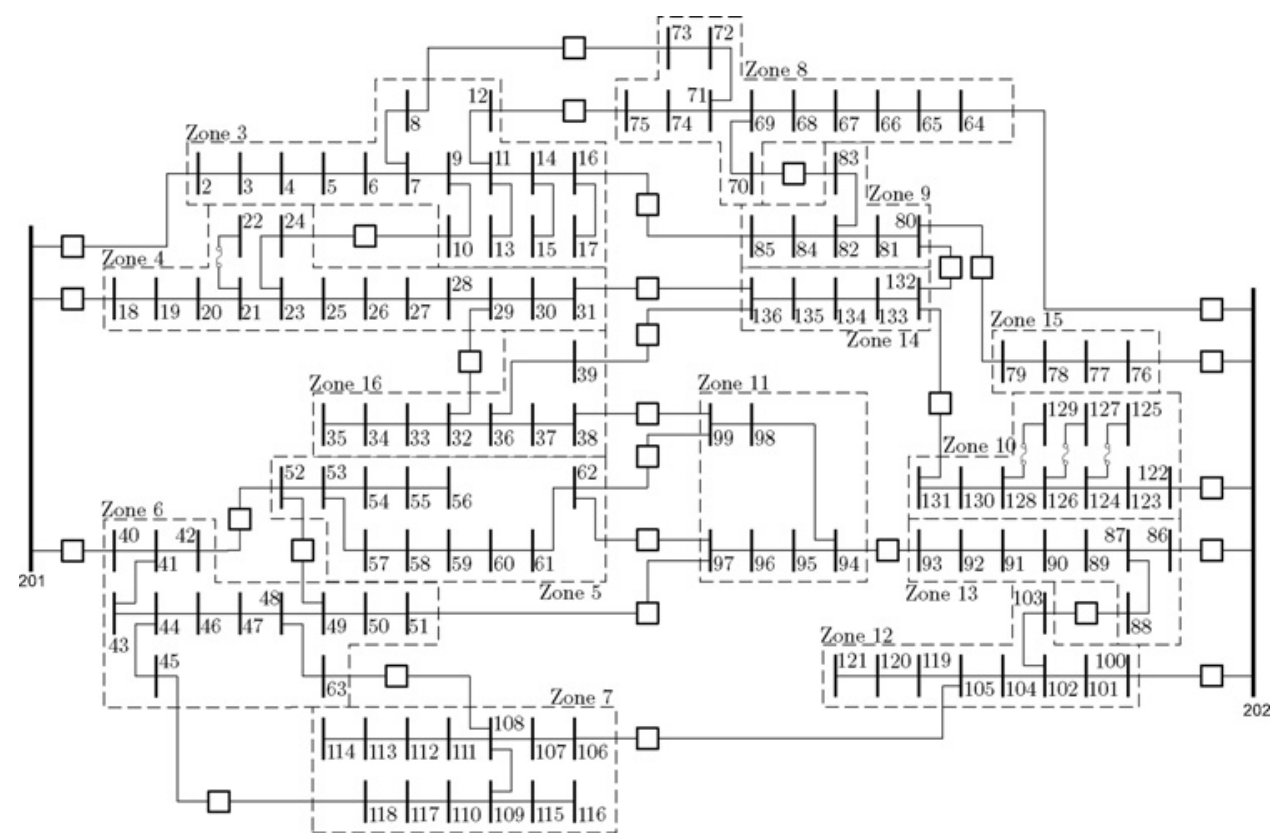

Fig. 4 136-node real system with 16 load zones

$$
\begin{gathered}
\sum_{k \in \Omega_{b}} \sum_{z \in \Omega_{z}}\left[N_{k}\left(r_{z}-r_{\mathrm{sw}}\right) \lambda_{z} z_{\mathrm{act} k, z}+N_{k} r_{\mathrm{sw}} \lambda_{z} \omega_{z, k}\right]+2 z^{(U)} \Gamma \\
+\sum_{k \in \Omega_{b}} \sum_{z \in \Omega_{z}}\left(p_{z, k}^{(U), z_{\mathrm{act}}}+p_{z, k}^{(U), \omega}\right) \leq \sum_{k \in \Omega_{b}} N_{k}\left(\overline{\mathrm{SAIDI}}-\lambda_{\text {fused } k} r_{\hat{z}_{k}}\right) \\
z^{(\lambda)}+p_{z, k}^{(\lambda), \omega} \geq N_{k} \sigma_{z} \omega_{z, k} \quad \forall k \in \Omega_{b}, \forall z \in \Omega_{z} \\
z^{(U)}+p_{z, k}^{(U), z_{\mathrm{act}}} \geq N_{k}\left(r_{z}-r_{\mathrm{sw}}\right) \sigma_{z} z_{\mathrm{act} k, z} \quad \forall k \in \Omega_{b}, \forall z \in \Omega_{z} \\
z^{(U)}+p_{z, k}^{(U), \omega} \geq N_{k} r_{\mathrm{sw}} \sigma_{z} \omega_{z, k} \quad \forall k \in \Omega_{b}, \forall z \in \Omega_{z} \\
z^{(\lambda)} \geq 0 \quad \text { and } \quad z^{(U)} \geq 0 \\
p_{z, k}^{(\lambda), \omega} \geq 0, p_{z, k}^{(U), z_{\mathrm{act}}} \geq 0, p_{z, k}^{(U), \omega} \geq 0 \quad \forall k \in \Omega_{b}, \forall z \in \Omega_{z}
\end{gathered}
$$

Note that, new dual variables $z_{i}$ and $p_{i j}$ have been created in (50) and (56) for every binary reliability variables $z_{\text {act } k z}$ and $\omega_{z, k}$. Also, considering that the number of decision variables in (51) is twice than (50), parameter $\Gamma$ of (51) is multiplied by 2 . The use of only one parameter $\Gamma$ in the robust model is convenient because it makes possible to change the robustness of the model by adjusting only one $\Gamma$ parameter. Moreover, $0 \leq \Gamma \leq\left|\Omega_{z}\right|\left|\Omega_{b}\right|$. Equations (27)-(38), used to determine the average value of each failure and duration rates, are considered deterministic, so they are not affected by the robust formulation.

\section{MISOCP for the robust reconfiguration of EDS with reliability constraints}

Finally, the MISOCP for the optimal reconfiguration of EDS considering reliability indices assessment and robust reliability constraints is given by the following equation

$$
\left\{\begin{array}{l}
\min \quad c^{\mathrm{lss}} \sum_{i j \in \Omega_{l}} R_{i j} I_{i j}^{\mathrm{sqr}} \\
\text { s.t. } \\
\text { (7), (9), (11)-(23), (27)-(41), (50)-(56) }
\end{array}\right.
$$

\section{Tests and results}

The 136-node real system shown in Fig. 4 was used to demonstrate the performance of the proposed model. Electrical and reliability data can be obtained in [31]. The constants of the system are $c^{\text {lss }}$ $=\$ 1 / \mathrm{kW}, V_{\text {nomf }-n}=7.967 \mathrm{kV} \underline{V}=0.9 \mathrm{~V}_{\text {nom }}, \bar{V}=1.1 \mathrm{~V}_{\text {nom }}, r_{\mathrm{sw}}=$ $0.5 \mathrm{~h},\left|\Omega_{z}\right|=18$ and $\left|\Omega_{b}\right|=138$. Reliability limits were set at $\overline{\text { SAIDI }}=5.24 \mathrm{~h} /$ cust $/ \mathrm{yr}$ and $\overline{\mathrm{SAIFI}}=4 \mathrm{int} / \mathrm{cust} / \mathrm{yr}$, as established by the Brazilian EDS regulator in [32]. The proposed model was implemented in AMPL [21] and solved with CPLEX [22], (with default options and with a maximum gap of $1 \%$ as optimality criterion) on a workstation with an Intel Core i5-4570 processor.

The 136-node system in Fig. 4 has two source nodes, corresponding to nodes 201 and 202, and 16 load zones highlighted with dashed lines. Switches in Fig. 4 are indicated with squares, and some lateral branches are protected with fuses. Table 1 contains the average failure rates $\left(\lambda_{z}\right)$, average restoration times $\left(r_{z}\right)$ and standard deviations $\left(\sigma_{z}\right)$ of each zone, calculated using (24), (25) and (6), respectively.

To obtain all the feasible robust solutions of (57), parameter $\Gamma$ is iteratively adjusted as follows:

(i) Let $k \leftarrow 0$. Set $\Gamma=0$, and solve (57) robust MISOCP model. (ii) Save $\mathrm{SAIDI}_{k}, \mathrm{SAIFI}_{k}$ and the operating point obtained in the $k$ th iteration.

(iii) Set $k \leftarrow k+1, \Gamma=k$ and solve (57) robust MISOCP model. If feasible, return to step ii; otherwise, continue to step 4.

(iv) Display all feasible robust solutions.

All feasible robust solutions for the 136-node system found using the former procedure are shown in Table 2 . All voltage and current

Table $1 \lambda_{z}, r_{z}$ and $\sigma_{z}$ for each zone of the 136-node system shown in Fig. 4

\begin{tabular}{lccccccc}
\hline Zone & $\begin{array}{c}\lambda_{z} \text {, int/ } \\
\text { year }\end{array}$ & $r_{z}, \mathrm{~h}$ & $\begin{array}{c}\sigma_{z} \text { int/ } \\
\text { year }\end{array}$ & Zone & $\begin{array}{c}\lambda_{z} \text {, int/ } \\
\text { year }\end{array}$ & $r_{z}, \mathrm{~h}$ & $\begin{array}{c}\sigma_{z} \text { int/ } \\
\text { year }\end{array}$ \\
\hline 3 & 0.75 & 2.00 & 0.87 & 10 & 0.30 & 2.00 & 0.63 \\
4 & 0.60 & 2.00 & 0.77 & 11 & 0.25 & 2.00 & 0.50 \\
5 & 0.50 & 3.00 & 0.71 & 12 & 0.40 & 3.00 & 0.63 \\
6 & 0.60 & 2.00 & 0.77 & 13 & 0.35 & 2.00 & 0.59 \\
7 & 0.60 & 3.00 & 0.77 & 14 & 0.20 & 2.00 & 0.45 \\
8 & 0.55 & 2.00 & 0.74 & 15 & 0.15 & 2.00 & 0.39 \\
9 & 0.25 & 2.00 & 0.50 & 16 & 0.35 & 2.00 & 0.59 \\
\hline
\end{tabular}


Table 2 Configurations of the feasible robust solutions for the 136-node system

\begin{tabular}{|c|c|c|c|c|c|}
\hline$\Gamma$ & Topology (open switches) & $\begin{array}{l}\text { Power losses, } \\
\quad[\mathrm{kW}]\end{array}$ & $\begin{array}{l}\text { SAIDI, [h/ } \\
\text { cust/yr] }\end{array}$ & $\begin{array}{l}\text { SAIFI, [int/ } \\
\text { cust/yr] }\end{array}$ & $\begin{array}{c}\text { Robustness, } \\
\%\end{array}$ \\
\hline$[0,36]$ & $\begin{array}{c}49-52,105-106,12-75,16-85,31-136,39-136,38-99,62-99,62-97,51-97, \\
45-118,8-73,70-83,88-103,80-132,24-10\end{array}$ & 787.62 & 2.61 & 2.97 & 78.4 \\
\hline$[37,41]$ & $\begin{array}{c}12-75,16-85,31-136,39-136,38-99,62-99,62-97,45-118,63-108,8-73 \\
70-83,88-103,42-52,80-132,93-94,24-10\end{array}$ & 866.70 & 2.60 & 2.91 & 80.4 \\
\hline$[42,43]$ & $\begin{array}{c}49-52,105-106,12-75,16-85,31-136,39-136,62-99,62-97,51-97,45-118, \\
8-73,70-83,88-103,80-132,32-29,24-10\end{array}$ & 912.34 & 2.58 & 2.92 & 80.8 \\
\hline [44] & $\begin{array}{c}202-64,16-85,31-136,38-99,62-99,62-97,45-118,63-108,8-73,70-83 \\
88-103,42-52,80-132,93-94,32-29,24-10\end{array}$ & 1233.67 & 2.67 & 2.89 & 81.2 \\
\hline
\end{tabular}

limits are satisfied. The first column in Table 2 identifies the interval of $\Gamma$ established by the iterative adjustment. As $\Gamma$ increases, the level of robustness is enhanced. Note that the least robust solution is obtained by setting $0 \leq \Gamma \leq 36$, which corresponds to the configuration that optimally minimises the active power losses in the system (with a value of $787.62 \mathrm{~kW}$ ). On the other hand, the most robust solution is obtained by setting $\Gamma=44$, but the active power losses are increased to $1233.67 \mathrm{~kW}$.

Note that, feasibility is lost for $\Gamma>44$. This means that there is not a feasible solution that prevents (42) and (43) from being violated in all probable values of $\lambda_{z}$. Then, given the average values and the standard deviations of the failure rates in Table $1,100 \%$ reliability robustness is not achievable for this particular system. The fourth and the fifth columns in Table 2 identify the average SAIDI and SAIFI for every robust solution, calculated by (22) and (23). Both indices are shown in order to demonstrate the model's capacity to determine the average reliability indices of the EDS depending on the system's topology.

Since the purpose of the robust formulation is to maintain both reliability indices below their limits, the last column in Table 2 indicates the robustness for each solution. Equation (58) and a set of Monte Carlo simulations were executed to establish the probability of (42) and (43) from being violated if all the parameters $\lambda_{z}$ stochastically change during the real application of each robust solution considering the uncertainty characterisation of $\lambda_{z}$.

\subsection{Monte Carlo simulation}

The level of robustness of each solution produced by the MISOCP model in (57), is calculated as follows:

(i) Set $n \leftarrow 0$ and $x \leftarrow 0$. Save the binary variables $z_{\text {act } z, k}^{*}$ and $\omega_{z, k}^{*}$ generated by the solution of (57).

(ii) Based on (5)-(6), randomly generate a new set of annual failure rates for every zone as $\lambda_{z n} \leftarrow \operatorname{Poisson}\left(\lambda_{z}\right)$.

(iii) Using (36) and (37), recalculate the annual failure rate $\left(\Lambda_{k}\right)$ and annual interruption duration $\left(U_{k}\right)$ of every node, and determine the new average $\mathrm{SAIFI}_{n}$ and $\mathrm{SAIDI}_{n}$ using (22) and (23).

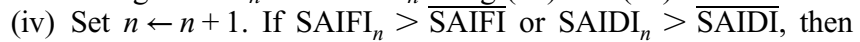
$x \leftarrow x+1$. Establish the robustness at iteration $n$ by using the following equation

$$
\text { Robustness }=\left(1-\frac{x}{n}\right) 100 \%
$$

(v) If convergence to the first decimal is achieved, then stop; otherwise, return to step ii.

Fig. 5 shows the process of convergence of the Monte Carlo simulations applied to every robust solution in Table 2. Each line in Fig. 5 represents the probability of each robust solution to maintain the system's SAIDI and SAIFI below the limits, even if parameters $\lambda_{z}$ randomly changes.

As shown in Table 2, there is a tradeoff between improving the reliability robustness and minimising the active power losses.
Therefore, the proposed methodology is an effective and accurate tool that utilities can use to reconfigure the EDS considering uncertainty in the reliability parameters, depending on their quality of service requirements.

\subsection{Reliability robustness versus average reliability optimisation}

The minimisation of both reliability indices, SAIDI and SAIFI, assuming the average value of $\lambda_{z}$, does not necessarily lead to robust solutions. To demonstrate this, the robust model in (57) is transformed into a non-robust weighted model given by (59). Note that, the robust reliability constraints (50)-(56) have been removed from (59). Both reliability indices are multiplied by a big positive number $M$ in order to enhance their relative weights in the objective function

$$
\left\{\begin{array}{l}
\min c^{\mathrm{lss}} \sum_{i j \in \Omega_{l}} R_{i j} I_{i j}^{\mathrm{sqr}}+M(\mathrm{SAIDI}+\mathrm{SAIFI}) \\
\text { s.t. } \\
\text { (7), (9), (11)-(23), (27)-(41) }
\end{array}\right.
$$

The non-robust MISOCP model in (59) is convex and can be optimally solved by CPLEX [22]. Table 3 contains the solution of (59) for the 136-node system, using $M=9999$. A large value of $M$ has been chosen with the aim of forcing the model to improve the average reliability indices. The last column indicates the level of robustness using Monte Carlo simulations as indicated in Section 7.1. Note that, the solution in Table 3 has less robustness than the most robust solution obtained with (57) in Table 2. Therefore, reliability robustness is not guaranteed by the direct minimisation of the average reliability indices.

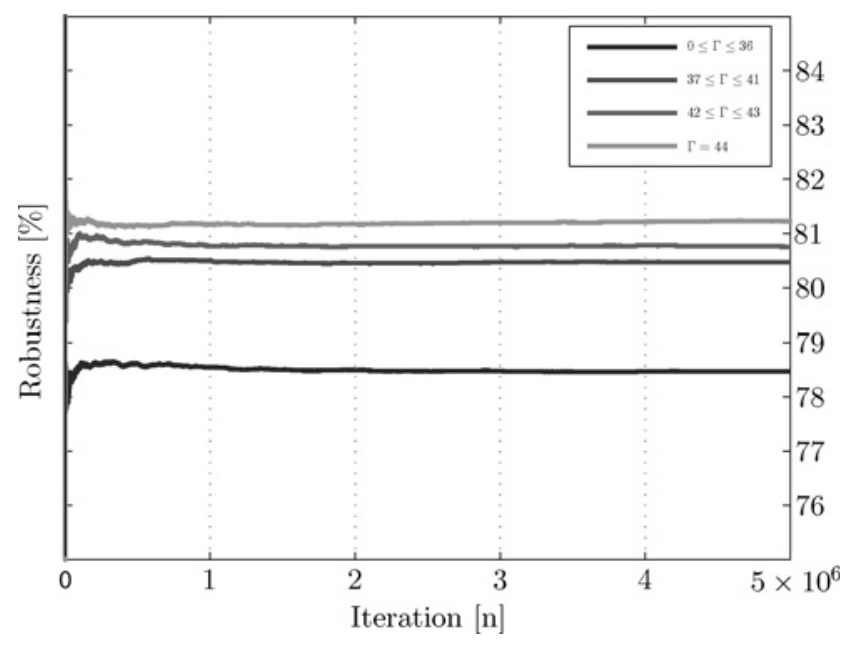

Fig. 5 Robustness assessment using Monte Carlo simulations 
Table 3 Solution of (59) applied to the 136-node system, $M=9999$

\begin{tabular}{lcccc}
\hline $\begin{array}{l}\text { Topology (open } \\
\text { switches) }\end{array}$ & $\begin{array}{c}\text { Power } \\
\text { losses, } \\
\mathrm{kW}\end{array}$ & $\begin{array}{c}\text { SAIDI, } \\
\text { [h/cust/ } \\
\mathrm{yr}]\end{array}$ & $\begin{array}{c}\text { SAIFI, } \\
\text { [int/cust/ } \\
\text { yr] }\end{array}$ & $\begin{array}{c}\text { Robustness, } \\
\%\end{array}$ \\
\hline $\begin{array}{l}12-75,16-85,31-136, \\
39-136,38-99,62-99,\end{array}$ & 866.7 & 2.60 & 2.91 & 80.4 \\
$62-97,45-118,63-108$, & & & & \\
$8-73,70-83,88-103$, & & & & \\
$42-52,80-132,93-94$, & & & & \\
$24-10$ & & & & \\
\hline
\end{tabular}

\section{Conclusions}

In this paper, a new MISOCP model was proposed to solve the reconfiguration problem of EDS considering the minimisation of active power losses and the robust improvement of the reliability indices, SAIDI and SAIFI. The proposed MISOCP model is a flexible, realistic and precise mathematical approach with convergence to optimality guaranteed by convex optimisation tools.

Uncertainty of the failure rates is a major concern in reliability assessment. As such, this paper presented a robust model that guarantees a reliable operation, even when uncertain reliability parameters change randomly during the real application of the solution. Finally, using Monte Carlo simulations, it has been shown that the model is accurate and that a tradeoff exists between the system topology that improves reliability robustness and the one that minimises active power losses in EDS.

\section{References}

1 IEEE Std. 493-2007: 'IEEE Recommended Practice for the Designing of Reliable Industrial and Commercial Power Systems', 2013

2 IEEE Std. 1366-2012: 'IEEE Guide for Electrical Power Distribution Reliability Indices', 2004

3 Billinton, R., Allan, R.N.: 'Reliability evaluation of power systems' (Springer, New York, 1996, 2nd edn.), ISBN 978-0306452598

4 Merlin, A., Back, H.: 'Search for a minimal-loss operation spanning tree configuration in an urban power distribution system'. Proc. V Power Systems Computer Conf., Cambridge, UK, 1975, pp. 1-18

5 Tang, L., Yang, F., Ma, J.: 'A survey on distribution system feeder reconfiguration: Objectives and solutions'. Proc. Innovative 2014 Smart Grid Technologies - Asia, Kuala Lumpur, 2014, pp. 62-67

6 Hsiao, Y.-T.: 'Multiobjective evolution programming method for feeder reconfiguration', IEEE Trans. Power Syst., 2004, 19, (1), pp. 594-599, ISSN $0885-8950$

7 Mendoza, J.E., Lopez, M.E., Coello, C.A.C., et al.: 'Microgenetic multiobjective reconfiguration algorithm considering power losses and reliability indices for medium voltage distribution network', IET Gener. Transm. Distrib., 2009, 3, (9), pp. 825-840, ISSN 1751-8687

8 Bernardon, D.P., Garcia, V.J., Ferreira, A.S.Q., et al.: 'Multicriteria distribution network reconfiguration considering subtransmission analysis', IEEE Trans. Power Deliv., 2010, 25, (4), pp. 2684-2691, ISSN 0885-8977

9 Amanulla, B., Chakrabarti, S., Singh, S.N.: 'Reconfiguration of power distribution systems considering reliability and power loss', IEEE Trans. Power Deliv., 2012, 27, (2), pp. 918-926, ISSN 0885-8977

10 Vitorino, R., Jorge, H., Neves, L.: 'Loss and reliability optimization for power distribution system operation', Electr. Power Syst. Res., 2013, 96, pp. 177-184, ISSN 0378-7796

11 Kavousi-Fard, A., Akbari-Zadeh, M.-R.: 'Reliability enhancement using optimal distribution feeder reconfiguration', Neurocomputing, 2013, 106, pp. 1-11, ISSN 0925-2312

12 Kavousi-Fard, A., Niknam, T. 'Multi-objective stochastic distribution feeder reconfiguration from the reliability point of view', Energy, 2014, 64, (0), pp. 342-354, ISSN 0360-5442

13 Kavousi-Fard, A., Niknam, T.: 'Optimal distribution feeder reconfiguration for reliability improvement considering uncertainty', IEEE Trans. Power Deliv., 2014, 29, (3), pp. 1344-1352, ISSN 0885-8977

14 Esmaeilian, H.R., Fadaeinedjad, R., Attari, S.M.: 'Distribution network reconfiguration to reduce losses and enhance reliability using binary gravitational search algorithm'. Proc. of the 22nd Int. Conf. and Exhibition on Electricity Distribution, Stockholm, 2013, pp. 1-4

15 Alonso, F., Oliveira, D., Zambroni de Souza, A. 'Artificial immune systems optimization approach for multiobjective distribution system reconfiguration', IEEE Trans. Power Syst., 2015, 30, (2), pp. 840-847, ISSN 0885-8950

16 Gupta, N., Swarnkar, A., Niazi, K.: 'Distribution network reconfiguration for power quality and reliability improvement using genetic algorithms', Int. J. Electric. Power Energy Syst., 2014, 54, (0), pp. 664-671, ISSN 0142-0615

17 Narimani, M.R., Vahed, A.A., Azizipanah, R., et al.: 'Enhanced gravitational search algorithm for multi-objective distribution feeder reconfiguration considering reliability, loss and operational cost', IET Gener. Transm. Distrib., 2014, 8, (1), pp. 55-69, ISSN 1751-8687

18 Carcamo-Gallardo, A., Garcia-Santander, L., Pezoa, J.: 'Greedy reconfiguration algorithms for medium-voltage distribution networks', IEEE Trans. Power Deliv., 2009, 24, (1), pp. 328-337, ISSN 0885-8977

19 Duan, D.-L., Ling, X.-D., Wu, X.-Y., et al.: 'Reconfiguration of distribution network for loss reduction and reliability improvement based on an enhanced genetic algorithm', Int. J. Electric. Power Energy Syst., 2015, 64, (0), pp. 88-95, ISSN 0142-0615

20 Bertsimas, D., Sim, M.: 'The price of robustness', Oper. Res., 2004, 52, (1), pp. 35-53, ISSN 0030-364X

21 Fourer, R., Gay, D.M., Kernighan, B.W.: 'AMPL: a modeling language for mathematical programming' (Brooks/Cole-Thomson Learning, Pacific Grove, CA, 2003, 2nd edn.), ISBN 978-0534388096

22 CPLEX Optimization Subroutine Library Guide and Reference, ILOG Inc., Incline Village, NV, CPLEX division edn., 2008

23 Cespedes, R.: 'New method for the analysis of distribution networks', IEEE Trans. Power Deliv., 1990, 5, (1), pp. 391-396, ISSN 0885-8977

24 Shirmohammadi, D., Hong, H.W., Semlyen, A., et al.: 'A compensation-based power flow method for weakly meshed distribution and transmission networks', IEEE Trans. Power Syst., 1988, 3, (2), pp. 753-762, ISSN 0885-8950

25 Lavorato, M., Franco, J.F., Rider, M.J., et al:: 'Imposing radiality constraints in distribution system optimization problems', IEEE Trans. Power Syst., 2012, 27, (1), pp. 172-180, ISSN 0885-8950

26 Romero-Ramos, E., Riquelme-Santos, J.: 'Discussion on 'imposing radiality constraints in distribution system optimization problems', IEEE Trans. Power Syst., 2013, 28, (1), pp. 568-568, ISSN 0885-8950

27 Romero-Ramos, E., Riquelme-Santos, J., Reyes, J.: 'A simpler and exact mathematical model for the computation of the minimal power losses tree', Electr. Power Syst. Res., 2010, 80, (5), pp. 562-571, ISSN 0378-7796

28 Borges, M.C.O., Franco, J.F., Rider, M.J.: 'Optimal reconfiguration of electrical distribution systems using mathematical programming, J. Control Autom. Electric. Syst., 2014, 25, (1), pp. 103-111

29 Franco, J.F., Rider, M.J., Romero, R.: 'A mixed-integer quadratically-constrained programming model for the distribution system expansion planning', Int. J. Electric. Power Energy Syst., 2014, 62, pp. 265-272, ISSN 0142-0615

30 Bazaraa, M.S., Jarvis, J.J., Sherali, H.D.: 'Linear programming and network flows' (John Wiley \& Sons, New York, 2009, 4th edn.), ISBN 978-0470462720

31136 Buses, 156 Branches Medium City Real Distribution System for Network Reconfiguration, URL http://www.feis.unesp.br/\#!/departamentos/engenhariaeletrica/pesquisas-eprojetos/lapsee/downloads/, 2015

32 Procedimentos de Distribuição de Energia Elétrica no Sistema Elétrico Nacional PRODIST. Módulo 8 - Qualidade da Energia Elétrica, Agência Nacional de Energia Elétrica, Braslia, DF, 2012

\section{Appendix}

The linear robust programming approach proposed by Bertsimas and Sim [20] is able to maintain model feasibility even if the uncertain parameters scholastically change without excessively deteriorate the objective function.

Consider the linear optimisation problem given by the following equation

$$
\left\{\begin{array}{l}
\min \sum_{j} c_{j} x_{j} \\
\text { s.t. } \\
\sum_{j} a_{i j} x_{j} \leq b_{i} \quad \forall i \\
l_{j} \leq x_{j} \leq u_{j} \quad \forall j
\end{array}\right.
$$

where $x_{j}$ is the set of decision variables, $c_{j}$ is the set of costs at the objective function and $a_{i j}$ is the set of coefficients for every constraint $i$ and variable $j$. Consider that $\Omega_{U i}$ contains all of the coefficients in the $i$ th constraint that are subject to uncertainty, and every uncertain parameter $a_{i j}, \forall j \in \Omega_{U i}$ is modelled as a random variable that takes values within the symmetric and bounded interval $\left[a_{i j}-\hat{a}_{i j}, a_{i j}+\hat{a}_{i j}\right]$, where $a_{i j}$ represents the mean value, and $\hat{a}_{i j}$ represents the maximum positive deviations around the mean value.

A new parameter $\Gamma_{i}$ for every constraint $i$ is introduced. $\Gamma_{i}$ controls the number of uncertain variables that can stochastically change without violating the $i$ th constraint. Thus, $\Gamma_{i}$ can only take values between 0 and $\left|\Omega_{U i}\right|$. With this in mind, the non-linear formulation in (60) is the mathematical model that makes possible to adjust the 
level of robustness of the final solution depending on the value of $\Gamma_{i}$.

$$
\left\{\begin{array}{l}
\min \sum_{j} c_{j} x_{j} \\
\text { s.t. } \\
\sum_{j} a_{i j} x_{j}+\max _{S_{i}\left|S_{i} \subseteq \Omega_{U i}\right| S_{i} \mid=\Gamma_{i}}\left\{\sum_{j \in S_{i}} \hat{a}_{i j}\left|x_{j}\right|\right\} \leq b_{i} \quad \forall i \\
l_{j} \leq x_{j} \leq u_{j} \quad \forall j
\end{array}\right.
$$

To linearise the formulation in (61), the term

$$
\beta_{i}\left(\boldsymbol{x}, \Gamma_{i}\right)=\max _{S_{i}\left|S_{i} \subseteq \Omega_{U i}\right| S_{i} \mid=\Gamma_{i}}\left\{\sum_{j \in S_{i}} \hat{a}_{i j}\left|x_{j}\right|\right\}
$$

is evaluated in a given solution $\boldsymbol{x}^{*}$ and transformed into a linear programming problem given by the following equation

$$
\left\{\begin{array}{l}
\beta_{i}\left(\boldsymbol{x}^{*}, \Gamma_{i}\right)=\max \sum_{j \in \Omega_{U i}} \hat{a}_{i j}\left|x_{j}^{*}\right| z_{i j} \\
\text { s.t. } \\
\sum_{j \in \Omega_{U i}} z_{i j} \leq \Gamma_{i} \\
0 \leq z_{i j} \leq 1 \quad \forall j \in \Omega_{U i}
\end{array}\right.
$$

Clearly, the solution of (62) determines the maximum possible deviation of the constraint $i$, if $\Gamma_{i}$ parameters are allowed to change. The dual model of (62) is given by (63) for every constraint $i$.

$$
\left\{\begin{array}{l}
\min \sum_{j \in \Omega_{U i}} p_{i j}+\Gamma_{i} z_{i} \\
\text { s.t. } \\
z_{i}+p_{i j} \geq \hat{a}_{i j}\left|x_{j}^{*}\right| \quad \forall i, \forall j \in \Omega_{U i} \\
p_{i j} \geq 0 \quad \forall j \in \Omega_{U i} \\
z_{i} \geq 0 \quad \forall i
\end{array}\right.
$$

Replacing the term $\beta_{i}\left(x, \Gamma_{i}\right)$ in (60) by the objective function of (63), and adding the new set of dual constraints, the non-linear robust formulation in (61) is transformed into the equivalent linear formulation shown in the following equation

$$
\left\{\begin{array}{l}
\min \sum_{j} c_{j} x_{j} \\
\text { s.t. } \\
\sum_{j} a_{i j} x_{j}+z_{i} \Gamma_{i}+\sum_{j \in \Omega_{U i}} p_{i j} \leq b_{i} \quad \forall i \\
z_{i}+p_{i j} \geq \hat{a}_{i j}\left|x_{j}\right| \quad \forall i, \forall j \in \Omega_{U i} \\
l_{j} \leq x_{j} \leq u_{j} \quad \forall j \\
p_{i j} \geq 0 \quad \forall i, \forall j \in \Omega_{U i} \\
z_{i} \geq 0 \quad \forall i
\end{array}\right.
$$

The formulation in (64) is a linear adjustable robust approach used to protect the original model from being unfeasible even when the uncertain parameters randomly change. 